\title{
Escuela y educación sobre desastres socionaturales en América Latina y el Caribe. Una revisión narrativa
}

\author{
School and Education on Socio-natural Disasters in Latin \\ America and the Caribbean. A Narrative Review \\ Escola e Educação sobre os Desastres Socionaturais na \\ América Latina e no Caribe. Uma Revisão Narrativa
}

Irene Villalobos Saldivia*, Adriana Espinoza Soto**

\section{RESUMEN}

Este artículo es una revisión narrativa, cuyo objetivo es identificar y caracterizar estudios realizados en América Latina y el Caribe acerca de la escuela y la educación en respuesta a desastres socionaturales. Se emplea la técnica de análisis temático a los artículos seleccionados de Clarivate, Web of Science, Scopus, Ebsco, SciELO, Redalyc, y repositorios de tesis. Los resultados se agrupan en dos ejes: 1) acciones educativas y desastres socionaturales, y 2) estrategias metodológicas utilizadas.

Las investigaciones contextualizan los desastres socionaturales dentro del proceso de formación escolar, enfatizando acciones para promover una cultura de prevención del riesgo y de autocuidado. Los estudios son en su mayoría cualitativos, estudios de casos de carácter participativo, que dan cuenta de la percepción del riesgo y de las iniciativas empleadas para enfrentar los desastres socionaturales. Se concluye desarrollar un enfoque holístico sobre el abordaje de emergencias y desastres socionaturales en comunidades educativas.
Palabras

clave: escuela, educación, desastres socionaturales, América Latina, El Caribe.

\footnotetext{
* Psicóloga, Universidad Arturo Prat. Magíster en Psicología Educacional, Universidad de Chile. Becaria ANID 2020, estudiante de Doctorado en Psicología, Universidad de Chile. Contacto: irene.v.saldivia@gmail.com

** Departamento de Psicología, Facultad de Ciencias Sociales, Universidad de Chile. Núcleo Investigación e Intervención en Emergencias y Desastres (NIID-UCHILE), Programa de Reducción de Riesgos y Desastres de la Universidad de Chile (CITRID). Contacto: adriana.espinoza@uchile.cl
} 


\begin{abstract}
This article is a narrative review whose objective is to identify and characterize studies carried out in Latin America and the Caribbean on schools and education in response to socio-natural disasters. The thematic analysis technique is used to selected articles from Clarivate, Web of Science, Scopus, Ebsco, Scielo, Redalyc, and thesis repositories. The results are grouped into two axes: 1. Educational actions and socio-natural disasters, and 2. The methodological strategies used.

The research contextualizes socio-natural disasters within the school training process, emphasizing actions to promote a culture of risk prevention and self-care. The studies are mostly qualitative, participatory case studies, which account for the perception of risk and the initiatives used to face socio-natural disasters. It is concluded to develop a holistic approach to dealing with emergencies and socio-natural disasters in educational communities.
\end{abstract}

\section{RESUMO}

Este artigo é uma revisão narrativa cujo objetivo é identificar e caracterizar os estudos realizados na América Latina e no Caribe sobre escola e educação em resposta a desastres sócio-naturais. A técnica de análise temática é usada para artigos selecionados do Clarivate, Web of Science, Scopus, Ebsco, Scielo, Redalyc e repositórios de teses. Os resultados estão agrupados em dois eixos: 1. ações educativas e desastres sócio-naturais, e 2. as estratégias metodológicas utilizadas.

A pesquisa contextualiza os desastres sócio-naturais no processo de formação escolar, enfatizando ações para a promoção de uma cultura de prevenção de riscos e autocuidado. Os estudos são, na sua maioria, estudos de caso participativos e qualitativos, que dão conta da percepção de risco e das iniciativas utilizadas para fazer face aos desastres socio-naturais. Conclui-se com o desenvolvimento de uma abordagem holística para lidar com emergências e desastres sócio-naturais em comunidades educacionais.
Key words: school, education, socionatural disasters, Latin America, Caribbean

Palavras-chave: escola, educação, desastres sócionaturais, América Latina, Caribe 


\section{Introducción}

Los desastres pueden ser causados por un hecho natural, como terremotos, tsunamis, erupciones volcánicas, huracanes y aluviones, o también ser producidos por condiciones biológicas (pandemias) o provocados por el ser humano (incendios o guerras) (Programa de las Naciones Unidas para el Desarrollo [PNUD], 2014). Las consecuencias de estos eventos afectan negativamente a las sociedades involucradas, porque perduran en el tiempo y, en ocasiones, provocan cambios sociales, económicos y medioambientales irreversibles. En especial, perjudican a aquellos territorios que presentan pobreza y vulnerabilidad estructural, puesto que éstos tienen una baja capacidad para anticipar, resistir y recuperarse ante estas situaciones (Banco Mundial, 2013; CEPAL, 2005).

Los desastres naturales, en todo el mundo, desde el año 2000 en adelante, han afectado a más de 2,7 billones de personas, las pérdidas económicas alcanzan 1,3 trillones de dólares y las humanas 1,1 millones. En 2010 el terremoto de Haití fue el más mortífero, con más de 220.000 víctimas fatales. En el terremoto de Japón de 2011 murieron alrededor de 20.000 personas y se registraron daños por $210.000 \mathrm{mi}-$ llones de dólares (González-Muzzio, 2013). En Latinoamérica, en los últimos 40 años se han registrado aproximadamente 310.000 fallecimientos y 30 millones de personas afectadas, con un costo de 213.000 millones de dólares a causa de los desastres naturales (CEPAL, 2014).

\section{Desastre socionatural}

La Organización Mundial de la Salud (OMS) señala que un desastre se define como una situación imprevista que representa serias e inmediatas amenazas para la salud, o cualquier situación de salud pública que pone en peligro la vida o salud de una cantidad significativa de personas y exige la acción inmediata (World Health Organization, 1990). Mientras que un comité de expertos de la Organización de Naciones Unidas (ONU, 1990) entiende de forma empírica los desastres como disrupciones (rupturas) del sistema ecológico humano, que sobrepasan la capacidad de respuesta de la comunidad afectada para poder abordar los efectos y funcionar con normalidad.

En la actualidad, la comunidad científica ha comenzado a utilizar el concepto de "desastre socionatural" para referirse a cualquier hecho 
concentrado en el tiempo y en el espacio en el que una sociedad, o una parte relativamente autosuficiente de ésta, vive un peligro severo, sufre pérdidas de sus miembros y deterioro de las condiciones materiales, debido a que la estructura social se rompe y todas o algunas de las funciones esenciales de la sociedad se ven impedidas (Cutter \& Emrich, 2006; Vargas, 2002; Campos, 1999; Fernández, Beristain y Páez, 1999). Esta conceptualización se centra en los efectos sociales más que en las características físicas de los desastres.

En América Latina y el Caribe el impacto de los desastres se agrava por las características propias de la región, como son los signos de inseguridad, incertidumbre y desprotección que se manifiestan en las esferas macro y microeconómicas, ambiental, social y cultural, las que derivan del modelo económico neoliberal implementado en la mayoría de los países, aumentando exponencialmente los riesgos (Ramos, 2000).

En términos psicosociales, la Organización Panamericana de la Salud (OPS, 2000) ha señalado que, cuando ocurre un desastre socionatural, las personas afectadas pocas veces tienden a presentar una conducta orientada hacia el pánico generalizado o la espera aturdida. Una vez que los sobrevivientes a tales eventos se recuperan del impacto situacional reaccionan con actitudes positivas. González-Muzzio (2013) señala que, con posterioridad al desastre, se incrementa la acción individual espontánea, pero sumamente organizada, para trabajar en comunidad en miras de la recuperación del entramado social que requiere de la ayuda de los gobiernos y, en muchos países, de recursos externos sin los cuales esto no sería posible.

Las consecuencias de los desastres y la pobreza inherente en Latinoamérica y el Caribe, según Arciniega (2014), no ha sido un obstáculo para superar adversidades. Bianchi (2011) estima que, además, la mayoría de las personas son capaces de recuperarse rápidamente después de una catástrofe, ya que en estas comunidades se da una condición particular que ha mejorado las habilidades colectivas para adaptarse en esta región donde cohabitan el riesgo y el peligro. Al respecto, Melillo y Suárez (2001) indican que, probablemente, esta capacidad de evaluar el evento positivamente está determinado por el desarrollo de la resiliencia, una variable que incrementa la habilidad individual para evitar las reacciones negativas o psicopatológicas a pesar de encontrarse en riesgo de generarlas. 


\section{La escuela y los desastres socionaturales}

La educación para los desastres, según Arauz-Muñoz (2008), constituye un concepto bastante nuevo, por lo que, en la década de los noventa, recién algunas personas se interesaron en la información científica y educación como principales componentes de la atención de emergencias y desastres. Al mismo tiempo, se puso énfasis en las etapas anteriores a la ocurrencia de un desastre, es decir, en la prevención, la mitigación y la preparación, y se consideró muy poco las etapas posteriores, como la ayuda humanitaria y la emergencia.

Del mismo modo, para Vélez (2012), la educación para desastres debe perseguir una formación en la cual las personas se involucren con nuevos saberes acerca de la naturaleza y con diversas áreas del ciclo vital humano - cognoscitiva, afectiva, ética y espiritual-, que permita no solo entender y conocer el entorno, sino también desarrollar capacidades y compromisos con el ambiente, la reducción de riesgos y la prevención de desastres.

En el desarrollo de la vida cotidiana, una de las principales funciones de la escuela es representar al sistema educativo formal para socializar a los sujetos en valores, actitudes y normas sociales (Dettmer, 2002; Vélez, 2011). Sin embargo, al estar frente a una situación de desastre, la escuela está llamada a articular otras dimensiones que permitan retomar la normalidad, movilizar sus estructuras e iniciar el proceso de reconstrucción social (Campos-González, 2017; Lillo, 2013; Vélez, 2011).

Desde organismos internacionales, como la Red Interagencial de Educación en Situaciones de Emergencia (2010, citado en Vélez, 2011), se afirma que la escuela, en su función educadora, aminora los impactos psicosociales de las catástrofes, pues otorga normalidad, estabilidad, estructura, y potencia, y se le otorga una perspectiva favorable con proyección en y para la sociedad. En este sentido, Denis (2014) enfatiza en la necesidad de educar como un esfuerzo permanente para la formación de nuevas percepciones y actitudes acerca del tema, no solo para responder a posibles desastres, sino también para combatir la vulnerabilidad existente, tanto en colegios como en territorios.

Diversos estudios y organismos internacionales han considerado que la escuela cumple una función articuladora en las distintas fases del desastre - preimpacto, impacto y postimpacto-, pues facilita a 
la población el acceso a nociones básicas y promueve el desarrollo de acciones colectivas, con el fin de salvaguardar la vida de las personas (Rossi, 1993, citado en Dettmer, 2002).

En general, se aprecia a la escuela como la institución promotora de una cultura de prevención. Sin embargo, ella no puede ser vista como un mero espacio de normalización ante este tipo de eventos. El problema que se configura sobre esta temática, según Campos-González (2017), Campos (1999) y Lillo (2013), es que existen pocos referentes entre la escuela y la comunidad en la fase de postimpacto de un desastre.

Por ello, surge el interés de recopilar antecedentes respecto de la escuela y la educación sobre desastres socionaturales, para elaborar una revisión que responda a ¿Qué se ha estudiado sobre la escuela y educación en contextos de desastres socionaturales en América Latina y el Caribe? A partir de esta pregunta se plantea el objetivo de identificar y caracterizar las investigaciones publicadas durante la última década respecto de la escuela y la educación sobre desastres socionaturales en América Latina y el Caribe.

\section{Metodología}

Se realizó una revisión narrativa de tipo cualitativa que, según Rother (2007), tiene como características principales describir y discutir teórica y/o contextualmente el "estado del arte" de una temática en particular. Según la autora, este tipo de revisiones de la literatura cumple un rol fundamental en la educación continua, porque permite adquirir y actualizar el conocimiento sobre un tema específico de manera relativamente rápida.

La recopilación de información se realizó con base en buscadores digitales de las siguientes revistas indexadas: Clarivate, Web of Science, Scopus, Ebsco, SciELO y Redalyc. Las dos primeras recogen producción internacional de alto impacto y confiabilidad científica, mientras que las restantes cuentan con amplitud de literatura de toda Latinoamérica. También se realizó la búsqueda en www.cybertesis.cl y www.cybertesis.net, repositorios que recogen este tipo de publicaciones académicas.

Para llevar a cabo la búsqueda de los artículos, los criterios de inclusión fueron los siguientes: 1) investigaciones empíricas relaciona- 
das con la escuela, educación y desastres socionaturales en América Latina o el Caribe; 2) año de publicación entre 2008 al 2019, y 3) idiomas español e inglés.

Los descriptores utilizados para la búsqueda resultaron de las siguientes combinaciones de palabras claves: escuela AND desastres socionaturales, escuela AND desastre ambiental, escuela AND zona de riesgo, escuela AND educación. Debido a que el descriptor "escuela" por sí solo amplia los resultados, entendiéndose como una institución pública en la que se imparte educación primaria y/o secundaria, se combinó mediante el operador booleano AND, para asegurar la búsqueda de contenido relacionada con desastres socionaturales y sus derivados pertinentes a esta revisión.

Al buscar las palabras claves, se eliminaron aquellos artículos y tesis que no tenían como tema central la escuela, la educación y los desastres socionaturales, en cualquiera de sus acepciones o definiciones. Luego de esta selección, se obtuvieron nueve artículos cualitativos y un artículo de metodología mixta. Estos estudios respetan los criterios de inclusión indicados para la presente revisión: seis en Chile, dos en México, uno en Costa Rica y uno en Cuba. Cabe destacar que uno de los criterios de inclusión de la búsqueda incluía idioma español o inglés, no se encontraron artículos en este último idioma.

Para registrar los datos, se organizó la información de cada artículo en los siguientes campos: autor(es), año, título, país, estrategia metodológica y un resumen de cada investigación. La tabla 1 presenta los estudios seleccionados.

Tabla 1

Características Descriptivas de Investigaciones sobre Escuelas y Desastres naturales

\begin{tabular}{|c|c|c|c|c|c|}
\hline Autor & Año & Título & País & $\begin{array}{l}\text { Estrategia } \\
\text { Metodológica }\end{array}$ & Resumen \\
\hline $\begin{array}{l}\text { Álvarez- } \\
\text { Gordillo, } \\
\text { G. D. C., } \\
\text { Álvarez- } \\
\text { Gordillo, L. } \\
\text { M., Eroza- } \\
\text { Solan, E. \& } \\
\text { Dorantes- } \\
\text { Jiménez, J.E. }\end{array}$ & 2008 & $\begin{array}{l}\text { Propuesta } \\
\text { educativa para la } \\
\text { gestión del riesgo } \\
\text { de desastres en la } \\
\text { Región Sierra de } \\
\text { Chiapas, México. }\end{array}$ & México & $\begin{array}{l}\text { Descriptivo, } \\
\text { diagnóstico } \\
\text { participativo. }\end{array}$ & $\begin{array}{l}\text { Confeccionan } \\
\text { un programa de } \\
\text { gestión de riesgo } \\
\text { para desastres } \\
\text { a través de } \\
\text { los resultados } \\
\text { obtenidos del } \\
\text { diagnóstico } \\
\text { participativo. }\end{array}$ \\
\hline
\end{tabular}




\begin{tabular}{|c|c|c|c|c|c|}
\hline $\begin{array}{l}\text { Izquierdo, } \\
\text { L. M. E. O., } \\
\text { Ramón, E. } \\
\text { G., Ruíz, M. } \\
\text { R. M. A. \& } \\
\text { Cortaza, M. } \\
\text { G. A. D. }\end{array}$ & 2010 & $\begin{array}{l}\text { Programa de } \\
\text { educación } \\
\text { ambiental, } \\
\text { prevención } \\
\text { y mitigación } \\
\text { de riesgos por } \\
\text { inundaciones } \\
\text { aplicado en } \\
\text { el Colegio de } \\
\text { Bachilleres de } \\
\text { Tabasco, Plantel } \\
\mathrm{N}^{\circ} 28 \text {. }\end{array}$ & México & $\begin{array}{l}\text { Descriptivo, } \\
\text { diagnóstico } \\
\text { participativo. }\end{array}$ & $\begin{array}{l}\text { Diseñan un } \\
\text { programa de } \\
\text { educación } \\
\text { ambiental a través } \\
\text { de los resultados } \\
\text { del diagnóstico } \\
\text { participativo. }\end{array}$ \\
\hline $\begin{array}{l}\text { Aguilar, M. \& } \\
\text { Brenes, G. }\end{array}$ & 2013 & $\begin{array}{l}\text { La percepción } \\
\text { de riesgo como } \\
\text { herramientas } \\
\text { para la gestión del } \\
\text { riesgo. Aportes } \\
\text { para la cogestión } \\
\text { comunitaria. } \\
\text { Caso de la } \\
\text { Comunidad de } \\
\text { Sixaola. Limón, } \\
\text { Costa Rica. }\end{array}$ & $\begin{array}{l}\text { Costa } \\
\text { Rica }\end{array}$ & $\begin{array}{l}\text { Descriptivo, } \\
\text { grupo focal y } \\
\text { entrevistas. }\end{array}$ & $\begin{array}{l}\text { Describen la } \\
\text { percepción del } \\
\text { riesgo como una } \\
\text { de las principales } \\
\text { herramientas para } \\
\text { trabajar la gestión } \\
\text { de riesgo en una } \\
\text { comunidad. }\end{array}$ \\
\hline $\begin{array}{l}\text { González- } \\
\text { Muzzio, C. }\end{array}$ & 2013 & $\begin{array}{l}\text { El rol del lugar y } \\
\text { el capital social } \\
\text { en la resiliencia } \\
\text { comunitaria } \\
\text { posdesastre. } \\
\text { Aproximaciones } \\
\text { mediante un } \\
\text { estudio de caso } \\
\text { después del } \\
\text { terremoto del } \\
\text { 27/F. }\end{array}$ & Chile & $\begin{array}{l}\text { Mixto: } \\
\text { cuantitativo } \\
\text { y cualitativo } \\
\text { descriptivo, } \\
\text { encuesta en línea } \\
\text { y entrevistas. }\end{array}$ & $\begin{array}{l}\text { Analiza el rol del } \\
\text { lugar y del capital } \\
\text { social en el periodo } \\
\text { de emergencia } \\
\text { posterremoto de } \\
2010 \text { en Chile. }\end{array}$ \\
\hline Denis, O. J. & 2014 & $\begin{array}{l}\text { La percepción } \\
\text { del riesgo como } \\
\text { componente } \\
\text { de la educación } \\
\text { ambiental en } \\
\text { las instituciones } \\
\text { escolares. }\end{array}$ & Cuba & $\begin{array}{l}\text { Descriptivo, } \\
\text { diagnóstico } \\
\text { participativo y } \\
\text { caracterización. }\end{array}$ & $\begin{array}{l}\text { Diseña un } \\
\text { programa de } \\
\text { educación } \\
\text { ambiental a partir } \\
\text { del diagnóstico y } \\
\text { caracterización } \\
\text { de la situación } \\
\text { ambiental del } \\
\text { entorno escolar. }\end{array}$ \\
\hline $\begin{array}{l}\text { Espinoza, A. } \\
\text { E., Espinoza, } \\
\text { C. E. \& } \\
\text { Fuentes, A. } \\
\text { A. }\end{array}$ & 2015 & $\begin{array}{l}\text { Retornando } \\
\text { a Chaitén: } \\
\text { diagnóstico } \\
\text { participativo de } \\
\text { una comunidad } \\
\text { educativa } \\
\text { desplazada por } \\
\text { un desastre } \\
\text { socionatural. }\end{array}$ & Chile & $\begin{array}{l}\text { Descriptivo, } \\
\text { diagnóstico } \\
\text { participativo. }\end{array}$ & $\begin{array}{l}\text { Elaboran un } \\
\text { proyecto, a partir } \\
\text { del diagnóstico } \\
\text { participativo, } \\
\text { para promover } \\
\text { herramientas } \\
\text { que permitan } \\
\text { consolidar una } \\
\text { comunidad } \\
\text { más resistente } \\
\text { a las amenazas } \\
\text { socionaturales. }\end{array}$ \\
\hline
\end{tabular}




\begin{tabular}{|c|c|c|c|c|c|}
\hline $\begin{array}{l}\text { Larenas, J. } \\
\text { A., Salgado, } \\
\text { M. A. \& } \\
\text { Fuster, X. S. }\end{array}$ & 2015 & $\begin{array}{l}\text { Enfrentar } \\
\text { los desastres } \\
\text { socionaturales } \\
\text { desde los } \\
\text { capitales } \\
\text { y recursos } \\
\text { comunitarios: } \\
\text { el caso de } \\
\text { la erupción } \\
\text { volcánica de } \\
\text { Chaitén, Chile. }\end{array}$ & Chile & Entrevistas. & $\begin{array}{l}\text { Analizan los } \\
\text { sistemas capitales } \\
\text { comunitarios y la } \\
\text { política pública de } \\
\text { gestión de riesgo } \\
\text { en la comunidad. }\end{array}$ \\
\hline $\begin{array}{l}\text { Castro, F. T., } \\
\text { Francisca; } \\
\text { Fava D. C. \& } \\
\text { Pérez, S. T. }\end{array}$ & 2015 & $\begin{array}{l}\text { Niños y niñas } \\
\text { como sujetos } \\
\text { sociales en la } \\
\text { gestión del riesgo: } \\
\text { estudio en tres } \\
\text { comunas con } \\
\text { diversos desastres } \\
\text { en Chile. }\end{array}$ & Chile & $\begin{array}{l}\text { Descriptivo, } \\
\text { mapas parlantes, } \\
\text { entrevistas } \\
\text { y cartas a } \\
\text { autoridades. }\end{array}$ & $\begin{array}{l}\text { Describen cómo } \\
\text { los niños y niñas } \\
\text { significan su } \\
\text { habitar en zonas } \\
\text { y localidades } \\
\text { afectadas por } \\
\text { siniestros o } \\
\text { catástrofes } \\
\text { socioambientales. }\end{array}$ \\
\hline $\begin{array}{l}\text { Berroeta, H., } \\
\text { Carvalho, L. } \\
\text { \& Di Masso, } \\
\text { A }\end{array}$ & 2016 & $\begin{array}{l}\text { Significados del } \\
\text { espacio público } \\
\text { en contextos de } \\
\text { transformación } \\
\text { por desastres } \\
\text { socionaturales. }\end{array}$ & Chile & $\begin{array}{l}\text { Descriptivo, } \\
\text { grupos focales } \\
\text { y análisis } \\
\text { discursivos. }\end{array}$ & $\begin{array}{l}\text { Analizan relatos } \\
\text { de personas } \\
\text { afectadas por tres } \\
\text { de los desastres } \\
\text { socionaturales que } \\
\text { ocurrieron en los } \\
\text { últimos años en } \\
\text { Chile. }\end{array}$ \\
\hline $\begin{array}{l}\text { Campos- } \\
\text { González, M. }\end{array}$ & 2017 & $\begin{array}{l}\text { Percepción social } \\
\text { del riesgo sísmico } \\
\text { en escuelas } \\
\text { de los barrios } \\
\text { patrimoniales } \\
\text { Yungay - Matta. }\end{array}$ & Chile & Entrevistas. & $\begin{array}{l}\text { Estudia la } \\
\text { percepción social } \\
\text { del riesgo en } \\
\text { escuelas de barrios } \\
\text { patrimoniales en } \\
\text { Chile. }\end{array}$ \\
\hline
\end{tabular}

Fuente: Elaboración propia.

\section{Resultados}

Se realizó un análisis temático (Barrera, Tonon y Alvarado, 2012) de los títulos, resúmenes y palabras claves de estos textos, identificando y caracterizando aspectos que han sido abordados por las escuelas de América Latina y el Caribe en la educación sobre desastres socionaturales. A partir de esta revisión, se clasifican de acuerdo con los temas que tratan, creando de manera inductiva dos ejes temáticos: 1) acciones educativas y desastres socionaturales, y 2) estrategias metodológicas utilizadas en las investigaciones. 


\section{1) Acciones educativas y desastres socionaturales}

Las investigaciones revisadas contextualizan los desastres socionaturales dentro del proceso de formación educacional. Se refieren a las escuelas y su relación con los riesgos socionaturales con el objeto de promover y potenciar acciones para una cultura de prevención, en miras de alcanzar políticas públicas idóneas según el contexto histórico y social de América Latina y el Caribe. Para otorgar una mayor comprensión de los antecedentes de esta categoría se agrupa la información en los siguientes temas: gestión del riesgo, experiencia del desastre y programas y/o proyectos psicoeducativos para la prevención del riesgo frente a desastre socionatural.

\section{Gestión del riesgo.}

Las investigaciones de Arauz-Muñoz (2008), Berroeta et al. (2016), Larenas, Salgado y Fuster (2015) y Vélez (2012) estiman que los desastres naturales son un tema actual y vigente, no solo por la magnitud y periodicidad de tales eventos que permiten visualizar el sentimiento de vulnerabilidad en la población, sino también por la apertura al debate sobre las condiciones estructurales, sociales e institucionales, y las formas de actuar y reaccionar de la población frente a las amenazas de la naturaleza.

Las comunidades de Latinoamérica y el Caribe se caracterizan por situarse en un territorio propenso a sufrir desastres socionaturales. Por ello, los estudios de Aguilar y Brenes (2013), Berroeta et al. (2016), Campos-González (2017), Castro, Fava y Pérez (2015), Espinoza, Espinoza y Fuentes (2015), González-Muzzio (2013) y Larenas et al. (2015) cuestionan las acciones tardías por parte del Estado. Por otro lado, la acumulación de experiencias de las comunidades ante estos eventos no ha permitido gestionar, por parte de las instituciones, abordajes futuros que apunten a la prevención y mitigación de riesgos frente a desastres, es decir, contar con una política pública que responda de manera oportuna a la población.

Las investigaciones de Aguilar y Brenes (2008), Berroeta et al. (2016), Campos-González (2017), Castro et al. (2015), Espinoza et al. (2015), González-Muzzio (2013) y Larenas et al. (2015) enfatizan en mejorar la eficacia, calidad y buena orientación de los Estados en la gestión de desastres, para propiciar la coordinación de todos los sec- 
tores, puesto que los efectos de estos eventos pueden ser disminuidos considerablemente si se adoptan medidas de prevención, reducción y mitigación de riesgos frente a desastres durante la formación escolar. Todo ello con el fin de disminuir la vulnerabilidad de las personas hasta lograr alcanzar una cultura de prevención, que se convierta en un referente para la comunidad y la ciudadanía.

Por otra parte, las investigaciones de Álvarez et al. (2008), CamposGonzález (2017), Denis (2014), Espinoza, et al. (2015) e Izquierdo, Ramón, Ruíz, y Cortaza (2010), concuerdan con un abordaje integral en el corto, mediano y largo plazo, puesto que buscan incorporar las características propias de cada comunidad en la formulación e implementación de programas y/o proyectos referidos a la prevención de riesgos sobre desastres socionaturales en las escuelas.

\section{Experiencia del desastre}

La propuesta de Álvarez et al. (2008), Berroeta et al. (2016), Castro et al. (2015) y Espinoza et al. (2015) busca conocer y analizar los relatos, representaciones sociales y significaciones de comunidades afectadas posdesastre, para explorar la configuración de los significados desde cómo enfrentan la catástrofe, hasta cómo han participado en el proceso cotidiano de toma de decisiones sobre su entorno, para reducir los riesgos en el proceso de transformación producidos por el desplazamiento o la reconstrucción social.

Por un lado, las investigaciones de Aguilar y Brenes (2013), Campos-González (2017) y Denis (2014) intentan posicionar a la percepción del riesgo como una de las principales herramientas para trabajar la gestión de este en la comunidad, debido a que permite conocer lo que las personas saben y entienden por "riesgo". Además, evalúan la forma en que se visualizan dentro del proceso de construcción en su propio riesgo para dotarse de herramientas que les permitan una mejor preparación para afrontar una posible situación de desastre. Por otro, el estudio de Izquierdo et al. (2010) presenta procedimientos para contribuir a la educación de la percepción de riesgos en los adolescentes, tomando como referencia la situación ambiental comunitaria y el modelo actual de la escuela. Según los resultados arrojados en el diagnóstico participativo realizado en este estudio, los adolescentes señalan interesarse en dicha temática; no obstante, carecen de cono- 
cimientos sobre la educación ambiental, específicamente en el ámbito de los desastres socionaturales.

\section{Programas y/o proyectos educativos para la prevención del riesgo de desastres socionaturales}

Los artículos de Aguilar y Brenes (2013), Álvarez et al. (2008) Castro et al. (2015), Espinoza et al. (2015), Denis (2014) e Izquierdo et al. (2010) desarrollan un análisis y reflexión sobre la relevancia de la educación para la prevención de riesgos socionaturales en el sistema educativo. Estas investigaciones sugieren que se debe impartir y promover una cultura de prevención, que otorgue nuevas condiciones de oportunidad a la escuela en el contexto posdesastre y prepare para los riesgos en la formación de ciudadanos instruidos en dichos eventos.

La mayoría de los estudios mencionados buscan elaborar, ejecutar y promover programas que se interrelacionen con la formación de conciencia ambiental y los desastres socionaturales, y, con ello, fomentar la participación de los miembros de la comunidad escolar y contribuir en la formación ciudadana, para que todas las personas sean capaces de sobreponerse frente a situaciones adversas y así transformar en una oportunidad el aprendizaje que poseen los miembros de la comunidad.

\section{2) Estrategias metodológicas de las investigaciones}

Respecto de las estrategias metodológicas empleadas por las investigaciones seleccionadas, cabe destacar que la gran mayoría son estudios cualitativos (Álvarez et al., 2008; Aguilar y Brenes, 2013; Berroeta et al., 2016; Denis, 2014; Espinoza et al., 2015; Izquierdo et al., 2010; CamposGonzález, 2017; Castro et al., 2015; Larenas et al., 2015), salvo el estudio de González-Muzzio (2013), que emplea una metodología mixta. Otro hallazgo relevante es que todas las investigaciones responden a estudios de casos, en los que se puede distinguir que seis utilizan entrevistas en profundidad, grupos focales y/o mapas parlantes (Aguilar y Brenes, 2013; Berroeta et al., 2016; Campos-González, 2017; Castro et al., 2015; González-Muzzio, 2013; Larenas et al., 2015), mientras que cuatro emplean diagnósticos participativos en la población de estudio, para la posterior implementación de programas y/o proyectos educativos sobre riesgos socionaturales (Álvarez et al., 2008; Denis, 2014; Espinoza et al., 2015; Izquierdo et al., 2010). 
Los estudios que utilizan grupos focales, entrevistas y/o mapas parlantes (Aguilar \& Brenes, 2013; Berroeta et al., 2016; Campos-González, 2017; Castro et al., 2015; González-Muzzio, 2013; Larenas et al., 2015), solo señalan la metodología utilizada pero no describen el procedimiento empleado durante la investigación.

Los estudios que utilizan el diagnóstico participativo (Álvarez et al., 2008; Denis, 2014; Espinoza et al., 2015; Izquierdo et al., 2010) concuerdan en que es la técnica que presenta mayor adaptación y flexibilidad frente a nuevas situaciones que surgen durante el proceso investigativo. Además, es una herramienta que permite mayor acercamiento a la comunidad y sus actores ya que, durante su aplicación, se recolecta un conjunto de antecedentes que permiten validar, modificar, contrastar o reformular los objetivos propuestos, para la posterior confección de programas, proyectos e intervenciones con relación a la prevención, reducción y mitigación sobre desastres socionaturales acorde a cada contexto educacional.

Para llevar a cabo el diagnóstico participativo, los estudios de Denis (2014) y Espinoza et al. (2015) trabajan con cada estamento educativo, estudiantes, padres y/o apoderados, docentes, funcionarios y asistentes de la educación, actividad en la que fue necesario desarrollar varias sesiones de talleres por cada grupo. El análisis de la información recolectada se efectuó a partir de la transcripción de los talleres y de las observaciones registradas en cada intervención. Los antecedentes recogidos fueron retroalimentados con los participantes, creando instancias de reflexión que llevaban a la identificación de sus propios recursos, así como al reconocimiento de nuevas necesidades con el fin de extrapolar el trabajo educativo a toda la comunidad.

Los artículos de Denis (2014) y de Izquierdo et al. (2010) utilizan el diagnóstico participativo con el objetivo de conocer y analizar el aprendizaje de los estudiantes en referencia a la educación ambiental en las instituciones escolares; mientras que el estudio de Álvarez et al. (2008) busca describir y analizar, desde la perspectiva de la comunidad, las representaciones sociales sobre su entorno, para reducir riesgos. Por otra parte, Espinoza et al. (2015) se centran en comprender, mediante esta técnica, la experiencia del desplazamiento y retorno de los diferentes actores educativos, el impacto en la salud mental de estos, así como sus necesidades y demandas laborales. 
Los estudios de Álvarez et al. (2008) y de Izquierdo et al. (2010) manifiestan que el análisis del diagnóstico participativo, integrado por los diversos sectores de la comunidad educativa, hacen posible el desarrollo de una investigación en acción participativa. Además, simultáneamente, se lleva a cabo la revisión bibliográfica de los abordajes sobre la gestión de riesgos de desastres socionaturales, con el fin de realizar acciones acordes con la población.

Todos estos estudios, una vez concluido el diagnóstico, cuentan con múltiples insumos para el diseño de programas, proyectos e intervenciones ante situaciones de desastres socionaturales y sobre la base de la educación, que sirven como una guía orientadora para la toma de decisiones y como complemento a otros trabajos que buscan incorporar o promover la cultura de prevención en la escuela.

\section{Discusión}

Para responder a la pregunta que guía el presente estudio sobre ¿qué se ha estudiado sobre la escuela y educación en contextos de desastres socionaturales en América Latina y el Caribe?, la discusión se agrupa en las siguientes categorías: 1) vivencia y percepción del riesgo, y 2) rol y capital social de la comunidad. Lo anterior, con el fin de facilitar la comprensión del contenido temático analizado.

\section{1) Vivencia y percepción del riesgo}

Las investigaciones de Aguilar y Brenes (2013), Álvarez et al. (2008), Berroeta et al. (2016), Castro et al. (2015), Denis (2014) y Espinoza et al. (2015) coinciden en señalar que las comunidades educativas estudiadas cuentan con diversas formas e interpretaciones sobre el riesgo socionatural. En este sentido, el estudio de Denis (2014) evidencia los distintos comportamientos frente a la percepción del riesgo, en lo fundamental a la concepción de los adolescentes sobre el medio ambiente, grupo etáreo en el cual se presentan carencias en las percepciones sobre las causas y consecuencias de los problemas ambientales identificados. Esto demuestra limitaciones en el tratamiento educativo en el proceso de formación integral. En esta línea, la investigación de Izquierdo et al. (2010) señala que más del $70 \%$ de estudiantes se muestra interesado en participar en un programa de educación ambiental de características activoparticipativas, puesto que las instituciones educativas no cuentan con tales programas. 
En las investigaciones de Álvarez et al. (2008), Campos-González (2017), Denis (2014), Espinoza et al. (2015) e Izquierdo et al. (2010) emerge un elemento transversal en las distintas etapas del estudio, debido a que los miembros de la comunidad educativa manifiestan una necesidad imperante de contar sus experiencias, ser escuchados y validados en su dolor por los investigadores, revelando la importancia de expresar sus vivencias entre los actores sociales, acción que los hace solidarizar y actuar de forma colaborativa los unos con los otros.

\section{2) Rol y capital social de la comunidad}

Los estudios de Álvarez et al. (2008), Aguilar y Brenes (2013) y Castro et al. (2015) expresan que los miembros de la comunidad tienen un conocimiento sobre el riesgo que enfrentan, pero este muchas veces no se comunica explícitamente, sino que se expresa en la vida cotidiana. Por lo tanto, la difusión de dicho conocimiento y experiencias acerca de los riesgos socionaturales permite la integración de agentes externos para participar en nuevos proyectos educativos.

Las investigaciones de González-Muzzio (2013), Espinoza et al. (2015) y Larenas et al. (2015) analizan la relación entre sistemas de capitales comunitarios (rol del lugar y capital social) y las políticas públicas de gestión del riesgo, con base en las narrativas y prácticas cotidianas de la comunidad. En este contexto, surgen nuevos grupos y comportamientos para fortalecer los aprendizajes relacionados con el enfrentamiento de riesgos socionaturales, lo que potencia el desarrollo continuo de la resiliencia comunitaria. De esta forma, se muestra por parte de la comunidad la capacidad de organizarse y comunicar el descontento con la autoridad, debido a que ésta no responde a sus necesidades mediante las acciones implementadas con posterioridad a un desastre socionatural.

Por otra parte, los resultados de los estudios de Álvarez et al. (2008), Berroeta et al. (2016), Espinoza et al. (2015), González-Muzzio (2013) y Larenas et al. (2015) estiman que las personas se adaptan rápidamente con sus propios recursos y promueven el desarrollo de nuevas habilidades en la comunidad, lo que permite identificar la identidad territorial y el sentido de pertenencia como elementos claves en la conformación de la resiliencia comunitaria. En consecuencia, los estudios de Berroeta et al. (2016), González-Muzzio (2013), Espinoza et al. (2015), 
Gordillo et al. (2008) y Larenas et al. (2015) plantean que las comunidades educativas son altamente cohesionadas y poseedoras de capital social, con fuerte sentido comunitario, lo que les ha permitido agilizar procesos de reconstrucción en sus comunidades.

\section{Conclusión}

La presente revisión narrativa tuvo como objeto de estudio identificar y caracterizar las investigaciones publicadas durante la última década respecto de la escuela y la educación sobre desastres socionaturales en América Latina y el Caribe. Esta da cuenta de estudios realizados en los siguientes países: Chile, Costa Rica, Cuba y México; por lo tanto, recopila información y aprendizajes que pueden ser relevantes para generar políticas públicas, tanto a nivel local como internacional y promover discusiones sobre programas, proyectos, protocolos y lineamientos de trabajo para el perfeccionamiento de estrategias educativas en las instituciones escolares.

Es interesante señalar que la mayoría de los artículos centra su mirada en todos los actores de la escuela, los cuales no solo son capaces de reflexionar y opinar respecto de sus experiencias, sino que son considerados como agentes claves por parte de los investigadores durante la recolección de la información, puesto que permiten ampliar la mirada de un mismo evento desde los distintos roles que desempeñan los participantes de los diversos estudios, relevando así importante información psicosocial, comunitaria y educativa. Además, las investigaciones informan acerca de las capacidades y recursos desplegados en las distintas dimensiones de la vida cotidiana que se ven afectadas por dichos eventos. A partir del reconocimiento de estas capacidades y recursos, se hace necesario fomentar y promover acciones comunitarias conjuntas entre los miembros de las comunidades afectadas y miembros de la academia, con el de elaborar proyectos y programas que permitan procesos de reconstrucción material y emocional en la etapa posdesastre.

En lo que se refiere a la vinculación de la escuela en el proceso estudiado, el primer elemento a destacar es la funcionalidad de mirar la vivencia del desastre como un modo de aproximarse a escudriñar sus efectos en las distintas etapas del ciclo vital humano. En segundo término, la escuela implica un importante apoyo psicosocial para toda 
la población, dado que todos los miembros de la comunidad escolar han experimentado algún fenómeno asociado a los desastres socionaturales y resulta ser el lugar en el que se entrega la mayor parte de los saberes socioculturales. Por eso, las investigaciones proponen la integración de conocimiento acerca de los desastres socionaturales en las distintas actividades curriculares de la escuela, puesto que permitiría a la sociedad sobrellevar con mejores recursos y herramientas estos eventos.

La información obtenida a través de esta revisión narrativa contribuye a nuevas interrogantes para futuras investigaciones respecto de las expectativas que surgen en torno al rol que desempeña la escuela y su comunidad educativa en contextos de desastres socionaturales. En retrospectiva, una limitación de este estudio fue optar por realizar una revisión narrativa sobre la escuela y la educación en respuesta a desastres socionaturales situándonos en América Latina y el Caribe, desestimando las experiencias en estas temáticas en otros continentes. No obstante, esta revisión presenta hallazgos que matizan la concepción de la escuela como un espacio de contención, normalización y expresión psicoeducativa de las emociones, precisando que esto lo logra una institución que es sentida como propia por parte de todos los miembros de la comunidad educativa y que es reconocida por la sociedad. Por esta razón, la escuela podría ser un eje central para desmitificar y democratizar el acceso a la información y al conocimiento como uno de los primeros pasos en la comprensión de los desastres socionaturales.

Otro aspecto fundamental sería la incorporación de estrategias pedagógicas para la prevención de riesgos, las cuales deben estar concentradas en las preocupaciones de los sujetos, en el conocimiento de su propio entorno y los saberes tradicionales, que deben ser considerados e incorporados como parte del proceso formativo. Por lo tanto, la recuperación de saberes trasmitidos entre generaciones contribuiría en la reducción de los efectos negativos de los desastres socionaturales, promoviendo de esta forma la participación comunal en los procesos educativos y, en general, en el desarrollo comunitario.

En otro ámbito, las acciones enmarcadas en el manejo del desastre por parte del Estado son reportadas en estas investigaciones como acciones tardías y que solo responden ante situaciones de emergen- 
cia (Aguilar y Brenes, 2013; Berroeta et al., 2016; Castro et al., 2015; Espinoza et al., 2015; González-Muzzio, 2013; Larenas et al., 2015). En consideración de estos antecedentes, se debe enfatizar en el carácter construido de los impactos de los desastres socionaturales, ya que son el resultado de las interacciones entre el medioambiente social, político y económico que estructura la vida de las personas en comunidad.

En esta misma línea, cabe destacar la importancia de las metodologías cualitativas de tipo participativo para la producción de conocimiento científico. Estas estrategias facilitan un acercamiento directo a las personas y comunidades afectadas, permitiendo de esta forma conocer sus experiencias, emociones y recursos desplegados en contextos de desastres socionaturales. Permiten también hacer un seguimiento a las acciones espontáneas y comprender cómo se configura la resiliencia en los grupos humanos, lo que constituye información altamente relevante tanto para estas comunidades como para los investigadores.

Entonces, es pertinente reforzar la producción de conocimientos científicos latinoamericanos, dada su escasa producción respecto de los impactos de los desastres socionaturales en las escuelas. Esto permitiría desarrollar temas incipientes en el ámbito educativo. En este sentido, los investigadores ponen énfasis en trabajar más allá de las intervenciones posdesastre y, además, avanzar en crear una cultura de prevención para la población.

Del mismo modo, se propone enfatizar la pertinencia de generar protocolos educativos para la capacitación y acompañamiento psicosocial de los miembros de la comunidad escolar. Todo lo anterior con el fin de colaborar con las políticas públicas y mejorar los proyectos, programas e intervenciones que se realizan por los entes gubernamentales, en el proceso de reconstrucción y reparación de daños posdesastres.

De igual manera, es necesario considerar en la organización y gestión educacional actividades de capacitación a toda la comunidad educativa y la integración de esta temática en textos y guías escolares. $\mathrm{Al}$ incluir los contenidos referidos a una cultura de prevención en el currículo, es posible influir significativamente en el modo de percibir, sentir, pensar, valorar y actuar de los estudiantes, respecto de todos los 
componentes y elementos que determinan la ocurrencia o no de una emergencia y/o desastre socionatural.

En consecuencia, los desastres socionaturales se presentan como un tema central para próximas investigaciones, dada la necesidad de educar y promover acciones que busquen disminuir el daño producido por diversos tipos de desastres, incluyendo aquellos que los mismos seres humanos ocasionan. Por lo tanto, los desafíos para América Latina y el Caribe son incorporar el diálogo con la sociedad civil, la comunidad científica y las instituciones educativas, para contribuir a la comunicación social sobre la base de las experiencias de estas comunidades.

Por último, es urgente desarrollar un nuevo enfoque educativo, que permita el análisis holístico de los desastres mediante la formación y capacitación, promoción y difusión informativa para posicionar dicha temática. La sociedad requiere de personas que, además de conocer los riesgos y las vulnerabilidades a los que están expuestos, sepan lidiar con ellos y tengan la capacidad de comprender y actuar apropiadamente ante situaciones de emergencias y desastres socionaturales.

\section{Referencias}

Aguilar, M. y Brenes, G. (2008). La percepción de riesgo como herramienta para la gestión del riesgo. Aportes para la cogestión comunitaria. Caso de la Comunidad de Sixaola. Limón, Costa Rica. Ponencia presentada en IX Congreso de Psicología Social para la Liberación. Chiapas, México.

Álvarez-Gordillo, G. D. C., Álvarez-Gordillo, L. M., Eroza-Solan, E. \& Dorantes-Jiménez, J. E. (2008). Propuesta educativa para la gestión del riesgo de desastres: en la región Sierra de Chiapas, México. Revista Mexicana de Investigación Educativa, 13(38), 919-943.

Arauz-Muñoz, J. (2008). Reflexiones sobre la educación de la prevención del riesgo a desastres en Costa Rica. Revista Tecnología en Marcha, 21(1), 202-214.

Arciniega, J. D. D. U. (2014). Resiliencia y envejecimiento. EJIHPE: European Journal of Investigation in Health, Psychology and Education, 4(2), 67-77. 
Banco Mundial. (2013). Informe sobre el desarrollo mundial 2014: riesgo y oportunidad: la administración del riesgo como instrumento de desarrollo.

Barrera, M. D. D., Tonon, G. y Alvarado, S. (2012). Investigación cualitativa: el análisis temático para el tratamiento de la información desde el enfoque de la fenomenología social. Universitas Humanisticas, 74(74).

Berroeta, H., Carvalho, L. y Di Masso, A. (2016). Significados del espacio público en contextos de transformación por desastres socionaturales. Revista INVI, 31(87), 143-170.

Bianchi, M. L. (2011). Impacto psicosocial de terremotos y tsunamis. Reflexiones basadas en investigaciones posteriores al 27-F chileno. Revista Sociedad, Ciudad y Territorio, 3.

Campos, A. (1999). Educación y prevención de desastres. Costa Rica, San José: UNICEF. http://www.flacsoandes.edu.ec/libros/10224opac

Campos-González, M. (2017). Percepción social del riesgo sísmico en escuelas de los barrios patrimoiales Yungay - Matta. Tesis para optar al grado de magister en Geografía, Universidad de Chile, Santiago, Chile.

Castro, F., Fava, D. y Pérez, S. (2015). Niños y niñas como sujetos sociales en la gestión del riesgo: estudio en tres comunas con diversos desastres en Chile. Seminario Internacional sobre Ciencias Sociales y Riesgo de Desastres: un encuentro inconcluso. Buenos Aires, 15 al 17 de septiembre.

CEPAL. (2005). Elementos conceptuales para la prevención y reducción de daños originados por amenazas socionaturales. Cuadernos de la CEPAL, 91, 1-68.

CEPAL, (2014). Naciones Unidas CEPAL. http://www.eclac.cl/

Cutter, S. \& Emrich, C. (2006). Moral hazard, social catastrophe: The changingface of vulnerability along the hurricane coast. Annals of the American Academy of Political and Social Science, 604, 102-112. http://www.jstor.org/stable/25097783

Denis, O. J. (2014). La percepción del riesgo como componente de la Educación Ambiental en las instituciones escolares. InfoCiencia, 18(4), 1-11.

Dettmer, J. (2002). Educación y desastres: reflexiones sobre el caso de México. Revista Latinoamericana de Estudios Educa- 
tivos (México), XXXII(2), 43-72. http://www.redayc.org/ pdf/270/27032203.pdf

Espinoza, A. E., Espinoza, C. E. y Fuentes, A. A. (2015). Retornando a Chaitén: diagnóstico participativo de una comunidad educativa desplazada por un desastre socionatural. Magallania (Punta Arenas), 43(3), 65-76.

Fernández, I., Beristain, C. y Páez, D. (1999). Emociones y conductas colectivas en catástrofes: ansiedad, rumor, miedo y conductas de pánico. En: J. Apalategui (Ed.). La anticipación de la Sociedad. Psicología Social de los movimientos sociales (pp. 281-342). Valencia: Promolibro.

González-Muzzio, C. (2013). El rol del lugar y el capital social en la resiliencia comunitaria posdesastre: Aproximaciones mediante un estudio de caso después del terremoto del 27/F. EURE (Santiago), 39(117), 25-48.

Izquierdo, L. M. E. O., Ramón, E. G., Ruíz, M. R. M. A. y Cortaza, M. G. A. D. (2010). Programa de educación ambiental, prevención y mitigación de riesgos por inundaciones aplicado en el Colegio de Bachilleres de Tabasco, Plantel no 28. Revista de la Alta Tecnología y la Sociedad. ISSN, 1940, 2171.

Larenas, J. A., Salgado, M. A. y Fuster, X. S. (2015). Enfrentar los desastres socionaturales desde los capitales y recursos comunitarios: el caso de la erupción volcánica de Chaitén, Chile. $M a$ gallania (Punta Arenas), 43(3), 125-139.

Lillo, M. (2013). Influencia de la reapertura de la escuela Juan José Latorre en el proceso de retorno de población desplazada por la erupción volcánica en Chaitén (tesis pregrado). Universidad de Chile, Santiago, Chile. http://repositorio.uchile.cl/handle/2250/130215

Melillo, A., y Suárez, E. (2001). Resiliencia, descubriendo las propias fortalezas. Buenos Aires: Paidós.

Organización Panamericana de la Salud. (2000). Los desastres naturales y la protección de la salud. Organización Panamericana de la Salud.

Organización de Naciones Unidas. (1989). International Decade for Natural Disasters Reduction. 44th Session of the General Assembly. Resolution 44/236. New York: ONU.

Programa de las Naciones Unidas para el Desarrollo [PNUD]. (2014). Informe sobre el Desarrollo Humano 2014. Sostener el Pro- 
greso Humano: Reducir las vulnerabilidades y construir resiliencia. https://www.undp.org/content/dam/undp/library/ corporate/HDR/2014HDR/HDR-2014-Spanish.pdf.

Ramos, R. (2000). Prometeo y las flores del mal: El problema del riesgo en la sociología contemporánea. Perspectivas sociológicas y principio de precaución.

www.proteccioncivil.org/ceise/ceisevirtual/ceisecv2000_ menu.htm

Rother, E. (2007). Revisión sistemática x revisión narrativa. Acta Paulista de Enfermagem, 20(2). Recuperado de http://www.scielo. br/pdf/ape/v20n2/es_a01v20n2.pdf

Vargas, J. (2002). Políticas Públicas para la reducción de la vulnerabilidad frente a los desastres naturales y socionaturales. CEPAL $N^{\circ}$ 50. Serie Medio Ambiente y Desarrollo. Santiago de Chile.

Vélez, B. (2011). La escuela en tiempos de crisis: Puntos de fuga para reinstaurar la esperanza en contextos post-desastres. Folios, (34), 25-35. http://revistas.pedagogica.edu.co/index.php/ $\mathrm{RF} /$ article/view/874/901

World Health Organization. (1990). European Regional Office. Emergency preparedness \& response program. EURO/EPR/90 Copenhage: WHO, European Regional Office. 